Article

\title{
Genome-Wide Identification and Transcriptional Expression of the METTL21C Gene Family in Chicken
}

\author{
Ge Yang ${ }^{\dagger}$, Hongzhao $\mathrm{Lu}^{\dagger}{ }^{\dagger}$, Ling Wang, Jiarong Zhao, Wenxian Zeng and Tao Zhang * \\ School of Biological Science and Engineering, Shaanxi University of Technology, Hanzhong, \\ Shaanxi 723001, China \\ * Correspondence: zl780823@163.com; Tel.: +86-091-6212-5002 \\ + These authors contributed equally to this work.
}

Received: 26 June 2019; Accepted: 15 August 2019; Published: 20 August 2019

\begin{abstract}
The chicken is a common type of poultry that is economically important both for its medicinal and nutritional values. Previous studies have found that free-range chickens have more skeletal muscle mass. The methyltransferase-like 21C gene (METTL21C) plays an important role in muscle development; however, there have been few reports on the role of METTL21C in chickens. In this study, we performed a genome-wide identification of chicken METTL21C genes and analyzed their phylogeny, transcriptional expression profile, and real-time quantitative polymerase chain reaction (qPCR). We identified 10 GgMETTL21C genes from chickens, 11 from mice, and 32 from humans, and these genes were divided into six groups, which showed a large amount of variation among these three species. A total of 15 motifs were detected in METTL21C genes, and the intron phase of the gene structure showed that the METTL21C gene family was conservative in evolution. Further, both the transcript data and qPCR showed that a single gene's (GgMETTL21C3) expression level increased with the muscle development of chickens, indicating that the METTL21C genes are involved in the development of chicken muscles. Our results provide some reference value for the subsequent study of the function of METTL21C.
\end{abstract}

Keywords: chicken; METTL21C; evolution; expression profiling

\section{Introduction}

The chicken (Gallus gallus domesticus) may originate from red junglefowl (G. gallus) [1]. Lueyang black-bone chicken is a famous native chicken breed with some distinctive features, such as its low fat content, strong muscles, and considerable nutritional and medicinal values. It has been listed in the Poultry Genetic Resources of China and was identified as a National Geographical Indication Product [2]. Chicken feeding methods are generally divided into caged and free range. It has been found that free-range chickens have more muscle mass [3]. A previous study showed that METTL21C is a skeletal-muscle-specific lysine methyltransferase [4]. It is well known that skeletal muscle plays an important role in the development of chickens, and can affect the athletic ability of the chickens. Thus, it is relevant to explore the role of the METTL21C gene in the muscle development of chicken species.

The methyltransferase-like 21C gene (METTL21C) belongs to the METTL21 family of the methyltransferase superfamily; in humans, METTL21C is located on 13q33.1 and is also called C13orf39 [5]. METTL21C, which has protein-lysine N-methyltransferase activity and participates in protein modification [6], is one of the protein methyltransferases (MTases) and exists in most eukaryotic organisms. It catalyzes the transfer of a methyl group from the cofactor S-adenosylmethionine (AdoMet) to a substrate and mainly occurs on lysine and arginine. MTases catalyze different biological reactions in organisms, which regulate many different substrates, such as lipids, RNA, DNA, small molecules, and proteins [7-9]. METTL21C, which performs regular protein structure housekeeping such as folding 
and proofreading, is also a $70 \mathrm{kDa}$ heat shock protein which acts as an ATP-dependent (Adenosine triphosphate dependent) molecular chaperone [10]. Further, bivariate genome-wide association studies (GWAS) have found that METTL21C can also act as a pleiotropic factor on muscles [11]. METTL21C can regulate calcium homeostasis in bones and muscles and promote the differentiation of myoblasts to myotubes through the nuclear factor (NF)-kB signaling pathway [11]. In addition, METTL21C may also reduce apoptotic osteocytes and regulate intracellular homeostasis [12]. A previous study concluded that METTL21C is an important regulator of protein degradation in skeletal muscles under normal and enhanced proteolytic conditions [4]. This suggests that METTL21C is closely related to the development of muscles and thus deserves further exploration.

The paralog of METTL21C is METTL21D, which is also a protein methyltransferase. Widely found in eukaryotes, METTL21D can catalyze the trimethylation of Lys315 in valosin-containing protein (VCP) in vitro, so it is also called VCP-KMT (valosin containing protein lysine methyltransferase) [6]. $\mathrm{VCP}$ is found in all eukaryotes and is a highly conserved AAA+ protein (ATPase associated with various cellular activities) [13]. It is a homotyped hexamer cyclic molecule comprising four domains: a flexible N-terminal domain and a short C-terminal domain, which may bind to the substrate, and two AAA ATP-binding domains [14-16]. VCP participates in a wide range of biological activities, including homotypic membrane fusion [17], endoplasmic reticulum-related degradation [18], DNA repair [19], and mitochondrial autophagy [20]. VCP and hereditary inclusion body myopathy are associated with Paget disease and frontotemporal dementia (inclusion body myopathy with early-onset Paget disease and frontotemporal dementia (IBMPFD)), which primarily affects muscles and the brain [21]. The pathogenesis of IBMPFD is due to the autosomal dominant single amino acid replacing the VCP terminal domain and the highly conserved residues within the D1 domain [21,22]. As mentioned above, VCP includes four domains; the domain D1 is one of the two AAA ATP-binding domains and so the mutation of the VCP gene is involved in the pathogenesis of IBMPFD [21]. In a recent study, it was found that METTL21D could promote tumor metastasis and affect cell growth, migration, and infection [23]. Since METTL21C is a homologous sequence of METTL21D, it was found that METTL21C interacts with VCP and catalyzes the modification and trimethylation of lysine 315 [4]. The METTL21C gene family from G. gallus domesticus offers an exceptional opportunity to study the evolution and function of muscle development systems [24]. In this study, to detect the mechanism of METTL21C in the development of chicken muscles, according the global genomic data on chickens, mice, and humans published by others, we systematically characterized 10 METTL21C genes of G. gallus, 11 METTL21C genes of Mus musculus, and 32 METTL21C genes of Homo sapiens. We used an iterative process of manual and computational analyses to identify examples of METTL21C in G. gallus, M. musculus, and H. sapiens encoding a gene family within the latest-released G. gallus, M. musculus, and H. sapiens whole-genome sequences [25-27]. We constructed a phylogenetic tree of the METTL21C gene family, the conserved motifs, gene structure, protein domain, and chromosome localization and further analyzed G. gallus METTL21C genes. To understand the function of the METTL21C genes in G. gallus, we also studied the transcriptional-level expression profiles of METTL21C of different tissues at different developmental stages. Our results provide useful theoretical support for the functional characterization of these METTL21C genes that are involved in the age-development process of G. gallus.

\section{Materials and Methods}

\subsection{Identification of METTL21C in Sequenced Genomes of G. gallus}

The query sequences of the METTL21C genes downloaded from the National Center for Biotechnology (NCBI) database (http://asia.ensembl.org/info/data/ftp/index.html) were for G. gallus, M. musculus, and H. sapiens. The whole protein sequence of G. gallus (http://asia.ensembl.org/info/ data/ftp/index.html?tdsourcetag=s_pcqq_aiomsg) was also downloaded from the NCBI database (http://asia.ensembl.org/info/data/ftp/index.html). Based on these sequences, using the BLASTP (basic 
local alignment search tool) software with E-value parameters less than 1e-10, a total of 22 genes were examined as the candidate sequence. To further check the members of the METTL21C gene family, we used a profile hidden Markov model (HMM) implemented with default parameters in HMMER v3.2.1 for Windows (http://hmmer.org/download.html/) according to the domain named pfam10007 [28].

\subsection{METTL21C Protein Alignment, Phylogenetic Analysis, Pfam Domain Detection, and Chromosome Location Analysis of G. gallus METTL21C Genes}

MEGA software was used to do a complete alignment based on the checked sequence with default settings [29], and an unrooted phylogenetic tree was constructed based on alignments using the neighbor-joining (NJ) method with pairwise deletion of 1000 bootstraps and a Poisson model. According to this phylogenetic analysis result, we classified these genes into different groups [30,31], and the conserved domains of these 119 METTL21C protein sequences were detected in Batch software (https://www.ncbi.nlm.nih.gov/Structure/bwrpsb/bwrpsb.cgi). Those domains found by Pfam were also detected by the SMART program (http://smart.embl-heidelberg.de/) with an E-value cutoff of 1.0 to validate the final result. Based on the GFF (general feature format) file, we anchored these genes to the related genomes through the MapChart software (http://mapchart.software.informer.com/2.2/) using the default parameters. The GFF file of these three species was downloaded from the NCBI database (https://www.ncbi.nlm.nih.gov/genome/?term=).

\subsection{Gene Structure and Motif Analysis of METTL21C Genes}

The exon-intron structure was illustrated using the online gene structure display server program (http://gsds.cbi.pku.edu.cn/) according to the information in the GFF file of the METTL21C gene family. The motifs were identified using the multiple EM (emphasize) for motif elicitation (MEME) program using the default parameters [32]. The parameters were as follows: the maximum number of motifs was set to 20, and the optimum motif width was set to 30-50.

\subsection{The Materials, Treatments, and Collections of Different Breeds of Chickens}

A total of six different breeds of black-bone chickens were collected, they included 20-day-old Liangfenghua chickens, 20-day-old Qingjiaoma chickens, 60- and 120-day-old Siyu chickens, and 60and 120-day-old Lueyang black-bone chickens. For each chicken development time, we considered periods of 20,60, and 120 days. We raised these chickens in cages or free-range: Lueyang black-bone chickens were free-range and the rest were caged. Different breeds of chickens had different feeding conditions. All the chickens are under normal circumstances, the optimal temperature for each stage of silky fowl through the brooding period (0 6 weeks) is as follows: $0 \sim 3$ days is $38 \sim 36{ }^{\circ} \mathrm{C}$, with a decrease of $2 \sim 3{ }^{\circ} \mathrm{C}$ every week to 6 weeks. The temperature should be gradually reduced by $21 \sim 18{ }^{\circ} \mathrm{C}$; the optimum temperature for broiler chickens after 6 weeks of age is $21 \sim 16^{\circ} \mathrm{C}$. The humidity in the house should be controlled by a hygrometer. The optimum suitable humidity for chicks is $55 \% \sim 65 \%$, and that of adult chickens is $60 \% \sim 70 \%$. Regarding the illumination, chicks of 1 to 3 days old maintain 23 to $24 \mathrm{~h}$ of light, 4 to 14 days of age maintain 16 to $19 \mathrm{~h}$ of light, and after 15 days of age, 8 to $9 \mathrm{~h}$ of light are maintained, and artificial light is applied when sunshine is insufficient. Free-range care generally does not require artificial control of the growing environment, and if necessary, some measures are taken according to the weather.

\subsection{Materials, Treatments, and Collection of the Transcriptome Profile of the METTL21C Gene Gamily of Chickens}

We put the samples in liquid nitrogen prior to storage at $-80{ }^{\circ} \mathrm{C}$ until use [33]. Total RNA was isolated using an RNA-prep Pure Kit (Tiangen, China) [34,35]. Libraries for RNA sequencing (RNA-seq) were produced using NEBNext Ultra RNA Library Prep Kit (NEB, Beverly, MA, USA). Paired-end sequencing was performed on the Illumina HiSeq2500 platform to generate $100 \mathrm{bp}$ reads using default parameters (Novogene Bioinformatics Technology Co., Ltd., Beijing, China (www.novogene.cn)). The 
de novo transcriptome was assembled using default settings in Trinity [36] and was based on the well-genome reference of chickens [37]. The assembled RNA-seq data were used to quantify these gene expression levels based on their fragments per kilobase of exon per million reads mapped (FPKM) values using Cufflinks with default parameters [38]. For each member's expression level of the METTL21C gene family, we used the HemI 1.0 software with default parameters [32,39]. For the expression level of several METT21C genes with error bars, the ggplot2 R package was used [40].

\subsection{Quantitative Real-Time PCR}

Because METTL21C is related to muscle development, we collected the skeletal muscle of different breeds at the same anatomic sites, such as the soleus muscle for the Siyu, Lueyang, Liangfenghua, and Qingjiaoma chickens. We also collected stomach, gizzard, lung, skeletal muscle, liver, skin, and myocardium from the Lueyang chickens (Table S1, Supplementary Materials). Total RNA was extracted from individual chicken tissues (stomach, gizzard, lung, skeletal muscle, liver, skin, and myocardium) and reverse transcribed to produce first-strand cDNA as described above. Real-time amplification reactions were performed on a 7500 fast real-time PCR system (Applied Biosystems, USA). The relative expression level of each gene was calculated according to the $2^{-\Delta \Delta C t}$ method [41]. The $\beta$-actin gene was used as an internal control [42], and all analyses were performed with three technical and three biological replicates (Table S2, Supplementary Materials).

\section{Results}

\subsection{Identification and Phylogenetic Analysis of METTL21C Genes}

To identify METTL21C-encoding genes, we seeded files for the lysine methyltransferase domains from G. gallus, M. musculus, and H. sapiens. We acquired 119 candidate METTL21C genes from these three species, including 22 G. gallus genes, $31 \mathrm{M}$. musculus genes, and $66 \mathrm{H}$. sapiens genes. To check for the presence of the lysine methyltransferase domain, those 119 candidate protein sequences were reduced to 53 genes that contain the same conserved domain as the lysine methyltransferase. Finally, we found 53 METTL21C genes in all species. In order to explore the evolutionary relationship among the members of the METTL21C families, an NJ tree based on G. gallus, M. musculus, and H. sapiens protein sequences was constructed (Figure 1). These three species had 10,11, and 32 METTL21C genes, respectively. Based on the completed alignment of the sequences and the phylogenetic analysis, we divided a total of 53 members of chickens, mice, and humans into six groups, named groups I to VI. (Table S3, Supplementary Materials). We found that group I contained 8 METTL21Cs, group II contained 10 METTL21Cs, group III contained 6 METTL21Cs, group IV contained 6 METTL21Cs, group V contained 5 METTL21Cs, and group VI contained 18 METTL21Cs. We found four G. gallus METTL21Cs (GgMETTL21C7, GgMETTL21C8, GgMETTL21C4, and GgMETTL21C3) to be closely associated with M. musculus, and six G. gallus METTL21Cs (GgMETTL21C9, GgMETTL21C5, GgMETTL21C1, GgMETTL21C2, GgMETTL21C6, and GgMETTL21C10) were found to be closely associated with H. sapiens. 


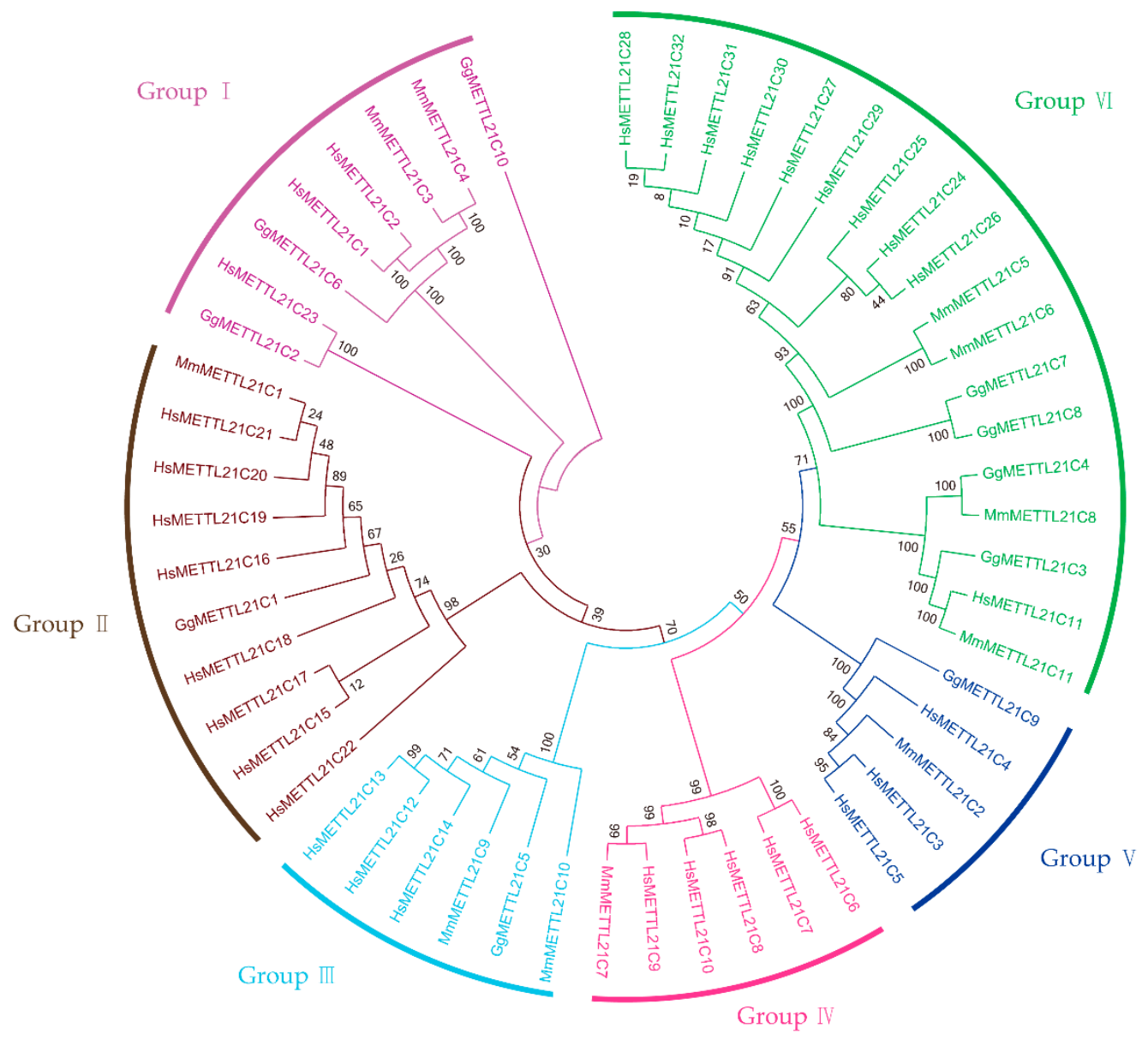

Figure 1. Phylogenetic analysis of methyltransferase-like 21C (METTL21C) proteins in G. gallus domesticus, M. musculus, and H. sapiens. These 53 sequences were used to construct a neighbor-joining (NJ) tree. The tree was divided into six groups (I-VI); the names of the different groups are displayed.

\subsection{Chromosomal Locations of METTL21C Genes and Their Relationship with G. gallus}

The 10 METTL21C genes were distributed unevenly in the G. gallus chromosomes based on the physical positions. We found the distribution of the METTL21C genes were different on each of the six chromosomes (Table 1; Figure 2). The accession number of the coding sequence and the genomic and protein IDs are listed in Table 1. Chromosomes 8 and 18 contained only one gene, GgMETTL21C1 and GgMETTL21C6, respectively, whereas GgMETTL21C3 and GgMETTL21C4 were both found on chromosome 1, but in different positions. GgMETTL21C2 and GgMETTL21C5, GgMETTL21C7 and GgMETTL21C8, as well as METTL21C9 and GgMETTL21C10 were found at different positions chromosomes chr14, chr7, and chr5, respectively (Figure 2). 
Table 1. Methyltransferase-like 21C (METTL21C) gene family information in chickens (G. gallus).

\begin{tabular}{|c|c|c|c|c|c|c|}
\hline \multirow{2}{*}{ Gene Name } & \multirow{2}{*}{ Protein ID } & \multirow{2}{*}{ CDS ID } & \multirow{2}{*}{ Gene ID } & \multirow{2}{*}{ Chromosome } & \multicolumn{2}{|c|}{ Gene Position } \\
\hline & & & & & Start & End \\
\hline GgMETTL21C1 & ENSGALP00000002756.3 & ENSGALT00000002759.6 & ENSGALG00000001790.6 & chr18 & $4,245,590$ & $4,247,698$ \\
\hline GgMETTL21C2 & ENSGALP00000003173.1 & ENSGALT00000003178.2 & ENSGALG00000002044.5 & chr14 & $13,793,041$ & $13,797,471$ \\
\hline GgMETTL21C3 & ENSGALP00000027192.5 & ENSGALT00000027243.7 & ENSGALG00000016864.7 & $\operatorname{chr} 1$ & $144,281,168$ & $144,285,163$ \\
\hline GgMETTL21C4 & ENSGALP00000027185.6 & ENSGALT00000027236.7 & ENSGALG00000016859.7 & chr1 & $144,171,934$ & $144,190,015$ \\
\hline GgMETTL21C5 & ENSGALP00000053406.2 & ENSGALT00000048292.2 & ENSGALG00000033603.3 & chr14 & $10,511,222$ & $10,519,988$ \\
\hline GgMETTL21C6 & ENSGALP00000047914.1 & ENSGALT00000056245.2 & ENSGALG00000035963.2 & chr8 & $5,470,226$ & $5,471,907$ \\
\hline GgMETTL21C7 & ENSGALP00000063245.1 & ENSGALT00000086206.2 & ENSGALG00000008493.7 & chr7 & $12,179,296$ & $12,182,133$ \\
\hline GgMETTL21C8 & ENSGALP00000032767.3 & ENSGALT00000033408.5 & ENSGALG00000008493.7 & chr7 & $12,179,725$ & $12,182,133$ \\
\hline GgMETTL21C9 & ENSGALP00000020014.7 & ENSGALT00000020041.7 & ENSGALG00000012269.7 & chr5 & $57,863,106$ & $57,866,374$ \\
\hline GgMETTL21C10 & ENSGALP00000073336.1 & ENSGALT00000098371.1 & ENSGALG00000051460.1 & $\operatorname{chr} 5$ & $57,862,387$ & $57,869,575$ \\
\hline
\end{tabular}

Note: Protein ID, gene ID, CDS ID, and gene position indicate that the accession numbers of the METTL21C gene family member sequences were downloaded from the National Center for Biotechnology (NCBI). CDS: Coding sequence. 


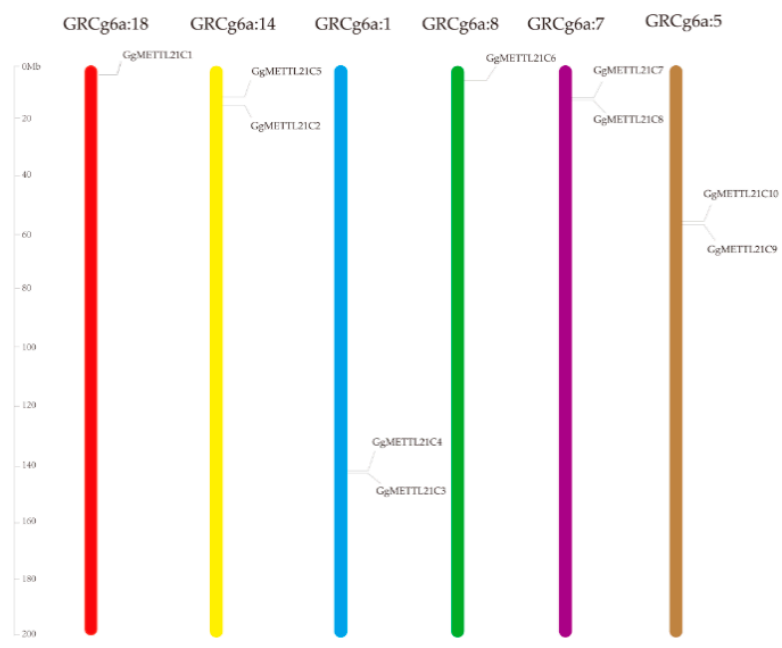

Figure 2. Chromosomal location in chickens of METTL21C genes on six chromosomes. Chromosomal locations in G. gallus are at the top of each bar. The scale of the chromosome is millions of base pairs $(\mathrm{Mb})$.

\subsection{METTL21C Gene Structures and Conserved Motifs}

To better understand the relationship between the structure and functions among METTL21C genes of G. gallus, M. musculus, and H. sapiens, the exon/intron organization and conserved motifs were analyzed and are shown in Figure 3. To compare the structural components of the 53 METTL21C genes, their structures, including exons and introns, were mapped. The maximum number of exons was found in MmMETTL21C9 (nine exons), and the minimum number of one exon was found in GgMETTL21C6. It seems that the most closely related members had similar numbers of exons. For example, those in Group I mostly had one exon, such as GgMETTL21C6, HsMETTL21C1, HsMETTL21C2, MmMETTL21C3, and MmMETTL21C4, but HsMETTL21C23 had six exons and GgMETTL21C10 had five exons. Members in Group II mostly had three exons, Group III mostly had nine exons, Group IV mostly had three exons, Group V had a varying number of exons (three to five), and Group VI mostly had three exons. Interestingly, we found there were one to nine exons in G. gallus, for example, GgMETTL21C6 had one exon, while GgMETTL21C5 had nine exons.

We also found using the MEME program that the METTL21C genes have 15 motifs. Motif 1 was mostly present in all breeds except GgMETTL21C7, HsMETTL21C7, HsMETTL21C14, HsMETTL21C22, and GgMETTL21C10. Motif 2 appeared in G. gallus, except GgMETTL21C9. As expected, the most closely related members in the same subfamilies shared a common motif composition, which may be indicative of similar function. These results suggest that all METTL21C genes in G. gallus share at least one typical domain. 
(a)

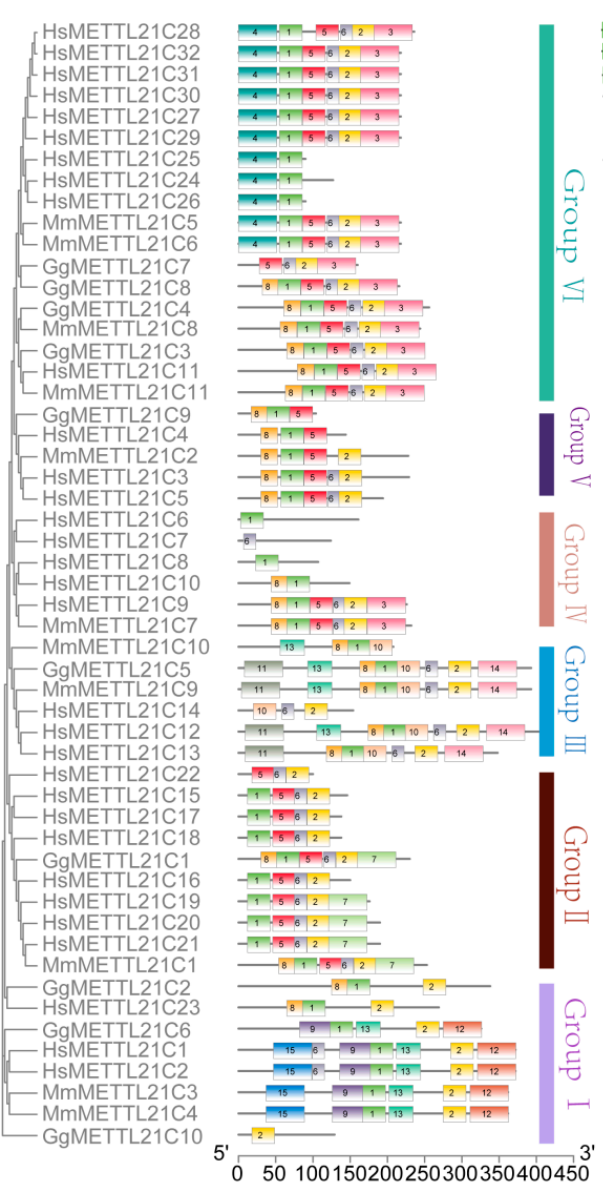

(c)

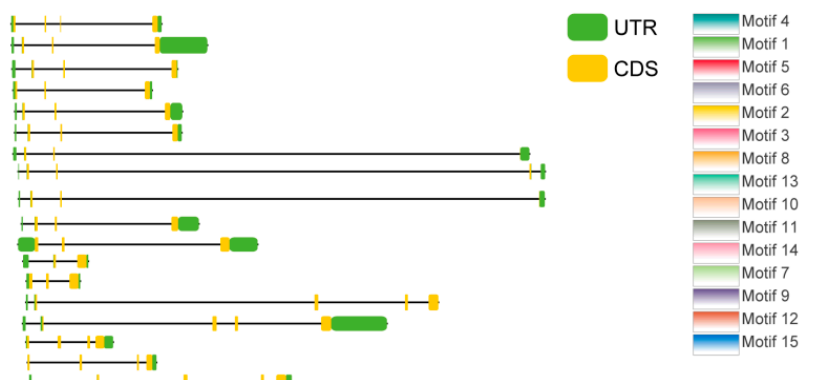

Figure 3. (a) Phylogenetic relationships, (b) motif compositions, and (c) gene structures of the 53 METTL21C genes identified in G. gallus (Gg), M. musculus (Mm), and H. sapiens (Hs). (a) Phylogenetic relationships using the $\mathrm{NJ}$ method, and different colors represent different groups. (b) Colored boxes indicate conserved motifs, and gray lines represent nonconserved sequences. The length of motifs in each protein is shown proportionally. (c) UTR represents upstream, CDS represents coding sequence, and EXON represents exon.

\subsection{Expression Profile Analysis of G. gallus METTL21C Genes}

To understand the expression patterns of different genes in the METTL21C gene family, we tested the expression levels of 10 genes in six samples from four breeds of chickens based on RNA-seq data (Table S4, Supplementary Materials). Based on phylogenetic analysis and expression levels, we divided the 10 members of chickens into three groups, named groups A, B, and C (Figure 4). Meanwhile, based on the different expression levels, we noticed that the expression levels of GgMETTL21C4, GgMETTL21C6, GgMETTL21C5, and GgMETTL21C10 were lowest and there was no difference in the different breeds and different development periods. Our results showed that GgMETTL21C7 and GgMETTL21C8 had relatively high expression in G. gallus, and there were some differences among the different samples. The expression patterns of GgMETTL21C7 and GgMETTL21C8 have breed specificity. Interestingly, we also detected that GgMETTL21C7, GgMETTL21C8, and GgMETTL21C2 genes had lower expression of Ly120 compared with Ly60. However, we found that GgMETTL21C3 had a large difference between chickens of different varieties. GgMETTL21C3 was highly expressed in Lueyang chickens and had a higher expression of Ly120 compared with Ly60. 


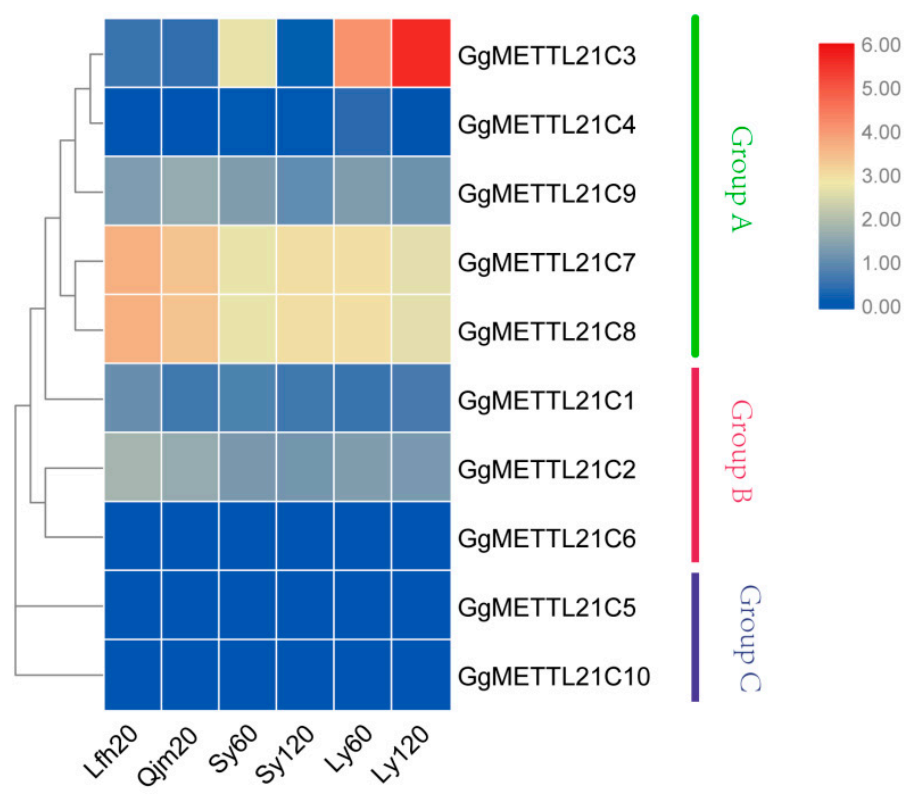

Figure 4. Expression patterns in METTL21C of G. gallus. The heat map was drawn in log10-transformed expression values. Red and blue represent relatively high and low expression, respectively, compared to the control. Lfh represents the chicken of Liangfenghua; Qjm represents the chicken of Qingjiaoma; Sy represents the chicken of Siyu; Ly represents the chicken of Lueyang. The numbers represent the age of the chickens in days.

\subsection{Real-Time PCR of METTL21C Genes of the METTL21C Gene Family}

These different breeds of chickens were not significantly different for growth in morphology, based on quantitative real-time PCR analysis of the expression of METTL21C3 in Sy and Ly chickens. The results showed that METTL21C3 was highly expressed in 120 days of Ly chickens (Figures 4 and 5a). Using $\beta$-actin as the internal reference gene, qPCR was used to detect the expression level of METTL21C3 in seven chicken tissues of Ly chickens, and it was found that the expression levels of myocardial and skeletal muscles were higher (Figure 5c). Based on quantitative real-time PCR analysis of the expression of METTL21C3 in soleus muscles and toe elongation muscles of Qjm and Ly chicken, we found that the expression level of METTL21C3 increased significantly with age (Figure 5d,e). Finally, we found that the expression level of METTL21C3 in free-range chickens was higher by comparing the expression levels of METTL21C3 in caged chickens and free-range chickens, further indicating that the METTL21C3 gene is indeed associated with muscle growth and development. 
(a)

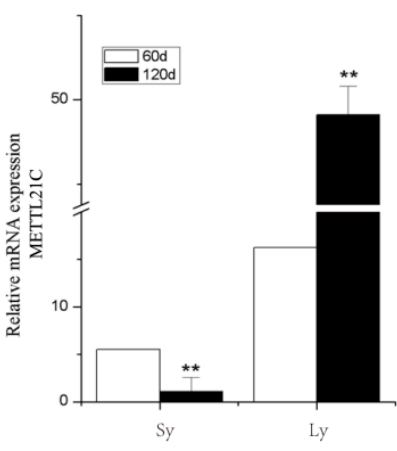

(c)

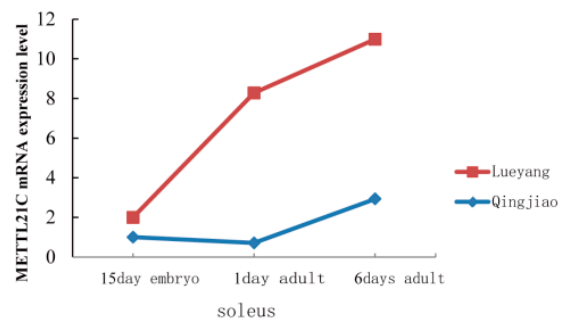

(e) (b)

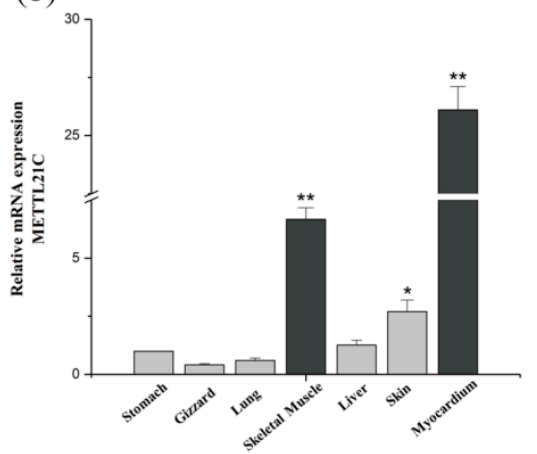

(d)

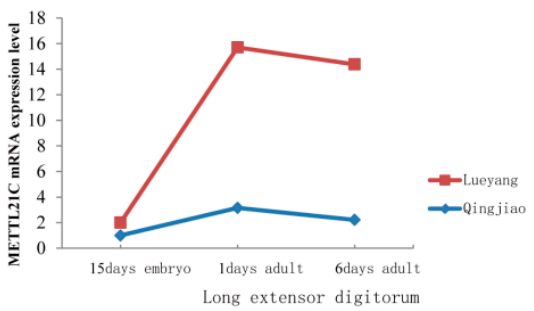

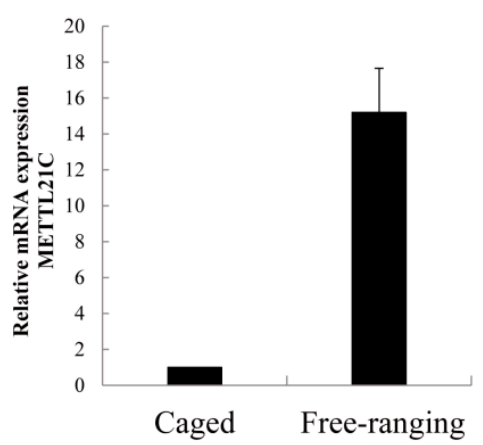

Figure 5. Relative mRNA expression: (a) METTL21C relative mRNA expression expression in different breeds of Sy and Ly chicken. (b) METTL21C relative mRNA expression in different tissues of Ly chicken. (c) METTL21C mRNA expression level in the soleus muscle. Lueyang represents Lueyang chickens and Qingjiao represents Qingjiao chickens. (d) METTL21C mRNA expression level in toe elongation muscles. (e) METTL21C relative mRNA expression in caged and free-ranging chickens. ${ }^{*} p \leq 0.05$; ** $p \leq 0.01$.

\section{Discussion}

\subsection{The Characteristics of the METTL21C Gene Family}

METTL21C is a skeletal-muscle-specific lysine methyltransferase which plays a critical role in the development of bones and muscles [4]. The METTL21C gene family is closely related to osteoporosis and sarcopenia by the NF-kB signaling pathway to prevent the differentiation of myoblasts [11]. The METTL21C genes of chickens contained three conserved domains identified in NCBI-Batch-CDD (NCBI Batch conserved domain database), including the AdoMet_MTases superfamily, SAM_superfamily, and FAM86, suggesting that METTL21Cs are extremely conservative in evolution (Figure S1). We found all chicken METTL21C genes had the conserved domain of AdoMet_MTases. The key domain is AdoMet_MTases (Figure S1), and in general, the most common methyl donor is S-adenosyl-L-methionine (AdoMet). The methylation reactions often occur in cells, 
which are catalyzed by MTases and induce methyl transfer from donor substrate molecules, implying that these genes have a similar function (Figure S1) [43]. In chickens, the number of domains of AdoMet_MTases is larger than that of other domains, such as the domain of SAM and FAM86.

The gene family is structurally and functionally similar and contains a common domain. For the METTL21C gene, the lysine methyltransferase domain was determined in a total of 10 member groupings in the METTL21C gene family. Based on phylogenetic analysis, the METTL21Cs are divided into six subfamilies named Groups I to VI. The present study identified 10 METTL21C genes in chickens, and more in M. musculus and H. sapiens (Table 1). The proteomes of these animals are different. For example, the M. musculus proteome is about $46,930 \mathrm{~kb}$, and the H. sapiens proteome is about 70,940 kb. However, the G. gallus proteome is about $25,209 \mathrm{~kb}$. Comparative analysis suggested that chickens, mice, and humans have similar numbers of gene families $[26,27,44]$, indicating that the number of METTL21Cs in different species is not determined by the genome size (Table 1).

In previous studies, GgMETTLC1 was called METTL23, which is a GABPA (GA-binding protein transcription factor, $\alpha$ subunit) function regulator that plays an important role in the transcriptional regulation of human cognition [45,46] and affects the nervous system and brain development [47]. Thus, we speculated that GgMETTL21C1 could be related to brain development in chickens. In terms of GgMETTL21C2, it contains the FAM86A protein that belongs to the protein MTases family, which catalyzes the trimethylation of eukaryotic elongation factor 2 (eEF2) on Lys-525 [48], indicating that the GgMETTL21C2 is involved in protein synthesis, which is critical for cell survival during stress [49]. In this study, GgMETTL21C3 was highly expressed in Lueyang chickens, compared to other breeds (Figures 4 and 5), and highly expressed in skeletal muscles compared to other muscles (Figure 5e). With the development of muscles in chickens, the expression level of GgMETTL21C3 is increased, these results show that GgMETTL21C3 is related to the development of skeletal muscles of Lueyang breeds (Figures 4 and 5a-d). In a previous study, Philippe referred to GgMETTL21C5 as METTL22, which can mediate methylation of kin17 on lysine 135. The kin17 is a DNA/RNA-binding protein that participates in the replication and repair of DNA and the process of pre-mRNA [50], and we found that GgMETTL21C5 plays an important role in chickens. In a previous study, Magnus found that METTL21A acts as a protein MTase, which is involved in the trimethylation of a conserved lysiner [51], this study also named the unnamed ID, which will enable others to identify the gene more clearly (Table S5, Supplementary Materials).

\subsection{The Evolution History of the METTL21C Gene Family of Chickens, Humans, and Mice}

Gene structure is an important factor for gene family evolution [52]. The location of the gene coding sequence and the structure of the protein are largely determined by the position of the intron, and protein diversity is further increased by exon shuffling and alternative splicing $[53,54]$. The association of the intron phase with conservation at splice site sequences is related to the evolution of spliceosomal introns. Intron phase 0 indicates the highest conservation, intron phase 1 also shows high conservation, and intron phase 2 shows the lowest conservation $[55,56]$. In the case of the METTL21C genes in chickens, almost all of the genes' structure was intron phase 0 , so the METTL21C genes were conservative in evolution (Figure 3c). In conclusion, based on these analyses, including motif and gene structure, we showed that METTL21C genes were conservative in evolution. We found that the METTL21C gene structure of chickens is different from that of humans and mice. The structure of METTL21Cs in chickens has undergone evolutionary changes (Figure 2c).

Conserved motif analysis revealed that almost all the METTL21C proteins contained one to five subdomains (Figure S1, Supplementary Materials). In addition, each subfamily had other special motifs, while some subfamilies also harbored specific motifs (Figure 3c). Interestingly, we found that the METTL21C genes of the same subfamily have a similar gene structure. This indicated that the gene structures are relatively conserved within each subfamily. For each subfamily, gene structures of METTL21C genes from chickens, mice, and humans are almost identical, suggesting that exon-intron structures of METTL21C genes are relatively conserved between these species. In particular, the 
GgMETTL21C10 gene has a long genetic distance, a simple genetic structure, and only one domain, probably because of the lack of structure during the evolution process (Figure 3). Its domain may be the AdoMet_MTases superfamily. Motif 1 existed in almost the entire METTL21C gene family of these three species, which means that the METTL21C gene family is conservative in evolution (Figure 3a).

\subsection{The Function of the METTL21C Gene Family of Chickens}

To detect the expression level of the METTL21C gene family members in different breeds of chickens, we sequenced six RNA-seq from different species at different periods. We then examined the expression level of METTL21C genes in different species of chickens (Table S1, Supplementary Materials). Based on the phylogenetic analysis and METTL21Cs' expression level, we divided these 10 genes into three groups. Group A included GgMETTL21C3, GgMETTL21C4, GgMETTL21C9, GgMETTL21C7, and GgMETTL21C8. Group B included GgMETTL21C1, GgMETTL21C2, and GgMETTL21C6. Group C included GgMETTL21C5 and GgMETTL21C10 (Figure 1). In previous studies, the METTL21C genes showed different expression patterns during different periods (20,60, and 120 days) in Lfh, Qjm, Sy, and Ly (Table S1, Supplementary Materials). However, there have been few reports on the METTL21C gene family in chickens. As the number of days increased, we found that the expression of METTL21C also increased, implying that these genes of METTL21C play a critical role in the development of chickens (Figure 4). The results showed that the METTL21C gene family can catalyze the development of bone and muscle [8]. Interestingly, GgMETTL21C7 and GgMETTL21C8 are in the same subfamily (Figure 1) and their expression was almost the same, indicating that these two genes are related to the growth and development of chickens [4]. Group $C$ had a low expression level (Figure 4). The expression level of METTL21C3 increased with the growth and development of chickens, further indicating that this gene may inhibit apoptosis and promote bone and muscle development (Figure 4) [4]. Importantly, the transcript data showed that, compared with Ly60, GgMETTL21C3 was highly expressed in Ly120, implying that GgMETTL21C3 plays an important role in the development of Lueyang species. Otherwise, by comparing the relative expression levels of METTL21C in Sy and Ly at 60 days and 120 days, it was found that GgMETTL21C was highly expressed in Ly at 120 days (Figure 5a); this evidence showed that GgMETTL21C3 is involved in the development of chickens. Therefore, we detected the relative expression of GgMETTL21C3 in different tissues of Ly chickens and found that the expression level was higher in skeletal muscle and myocardium (Figure 5b), further verifying that GgMETTL21C is related to skeletal muscle [4]. We also found that METTL21C is related to the growth and development of muscles in different breeds of chickens (Figure $5 \mathrm{a}, \mathrm{b}$ ) and in different feeding methods (Figure 5e). Both the transcript and qPCR analyses showed that GgMETTL21C3 is involved in the muscle development of Lueyang black-bone chickens, which is consistent with previous studies $[4,11,12]$.

\section{Conclusions}

In this study, we identified 10 METT21C genes in G. gallus. Phylogenetic analysis showed that the METTL21C genes can be divided into six groups based on specific and special domains. The 10 GgMETTL21Cs were respectively distributed on seven different chromosomes, with some colocalized to the same chromosome, such as GgMETTL21C7 and GgMETTL21C8 located in chromosome 7. Motif 1 was found in humans, mice, and chickens; Motifs 1, 5, 2, and 6 were found in most human HsMETTL21C genes; Motifs 1, 2, 5, and 8 were found in most mouse MuMETTL21C genes; and Motifs 1, 2, and 8 were found in most chicken GgMETTL21C genes. Based on this, we speculate that the conserved sequences are present in the METTL21C family. Expression profile analysis revealed that METTL21C gene family members display diverse expression patterns at different ages of different chickens. A total of three genes (GgMETTL21C3 GgMETTL21C7, and GgMETTL21C8) had high expression levels in G. gallus, suggesting they might play an important role in the development of chickens. In particular, the transcript data and qPCR analysis both showed that a single gene called METTL21C3 is involved in the development of different muscles of the Lueyang species, such as the soleus, long extensor digitorum, 
myocardium, and leg muscles. This study provides strong evidence that METTL21C3 may play a critical role in the development of chicken muscles.

Supplementary Materials: The following are available online at http://www.mdpi.com/2073-4425/10/8/628/s1, Figure S1: Conserved domain of the METTL21C family identified in 10 chickens (G. gallus). Table S1: A total of six samples of chicken muscles used for expression profiling in this study. Table S2: Primer information of qPCR. Table S3: The distribution numbers of METTL21C genes in Groups I-VI. Table S4: Expression data of METTL21C genes in flowers at different developmental stages of chickens (G. gallus). Table S5: Previous names of the METTL21C gene.

Author Contributions: Conceptualization, G.Y.; Data curation, H.L., L.W. and J.Z.; Funding acquisition, H.L. and T.Z.; Software, L.W. and W.Z.; Writing—original draft, G.Y., H.L. and T.Z.; Writing一review and editing, G.Y., H.L. and T.Z.

Funding: This study was supported by the Shaanxi Province Agricultural Science and Technology Innovation Project (2019NY-097) and the National Natural Science Foundation of China (NSFC) (31402071).

Acknowledgments: We thank Feng Yan, Ruiqing Li, and Jiarong Zhao for the sample collection.

Conflicts of Interest: The authors declare no conflict of interests.

\section{References}

1. Fumihito, A.; Miyake, T.; Sumi, S.; Takada, M.; Ohno, S.; Kondo, N. One subspecies of the red junglefowl (Gallus gallus gallus) suffices as the matriarchic ancestor of all domestic breeds. Proc. Natl. Acad. Sci. USA 1994, 91, 12505-12509. [CrossRef] [PubMed]

2. Zhang, T.; Liu, H.; Yang, L.K.; Yin, Y.J.; Lu, H.Z.; Wang, L. The complete mitochondrial genome and molecular phylogeny of Lueyang black-bone chicken. Br. Poult. Sci. 2018, 59, 618-623. [CrossRef] [PubMed]

3. Fleming, R.H.; Whitehead, C.C.; Alvey, D.; Gregory, N.G.; Wilkins, L.J. Bone structure and breaking strength in laying hens housed in different husbandry systems. Br. Poult. Sci. 1994, 35, 651-662. [CrossRef] [PubMed]

4. Wiederstein, J.L.; Nolte, H.; Günther, S.; Piller, T.; Baraldo, M.; Kostin, S.; Bloch, W.; Schindler, N.; Sandri, M.; Blaauw, B.; et al. Skeletal Muscle-Specific Methyltransferase METTL21C Trimethylates p97 and Regulates Autophagy-Associated Protein Breakdown. Cell Rep. 2018, 23, 1342-1356. [CrossRef] [PubMed]

5. Cloutier, P.; Lavallée-Adam, M.; Faubert, D.; Blanchette, M.; Coulombe, B. A Newly Uncovered Group of Distantly Related Lysine Methyltransferases Preferentially Interact with Molecular Chaperones to Regulate Their Activity. PLoS Genet. 2013, 9. [CrossRef] [PubMed]

6. Kernstock, S.; Davydova, E.; Jakobsson, M.; Moen, A.; Pettersen, S.; Maelandsmo, G.M.; Egge-Jacobsen, W.; Falnes, P.Ø. Lysine methylation of VCP by a member of a novel human protein methyltransferase family. Nat. Commun. 2012, 3. [CrossRef] [PubMed]

7. Martin, J.L.; McMillan, F.M. SAM (dependent) I AM: The S-adenosylmethionine-dependent methyltransferase fold. Curr. Opin. Struct. Biol. 2002, 12, 783-793. [CrossRef]

8. Schubert, H.L.; Blumenthal, R.M.; Cheng, X. Many paths to methyltransfer: A chronicle of convergence. Trends Biochem. Sci. 2003, 28, 329-335. [CrossRef]

9. Mosquera-Rendón, J.; Cárdenas-Brito, S.; Pineda, J.D.; Corredor, M.; Benítez-Páez, A. Evolutionary and sequence-based relationships in bacterial AdoMet-dependent non-coding RNA methyltransferases. BMC Res. Notes 2014, 7, 440. [CrossRef]

10. Mayer, M.P.; Bukau, B. Hsp70 chaperones: Cellular functions and molecular mechanism. Cell. Mol. Life Sci. 2005, 62, 670-684. [CrossRef]

11. Huang, J.; Hsu, Y.H.; Mo, C.; Abreu, E.; Kiel, D.P.; Bonewald, L.F.; Brotto, M.; Karasik, D. METTL21C is a potential pleiotropic gene for osteoporosis and sarcopenia acting through the modulation of the NF-kappaB signaling pathway. J. Bone Miner. Res. 2014, 29, 1531-1540. [CrossRef] [PubMed]

12. Zhao, F.; Gao, L.H.; Li, S.S.; Wei, Z.Y.; Fu, W.Z.; He, J.W.; Liu, Y.J.; Hu, Y.Q.; Dong, J.; Zhang, Z.L. Association between SNPs and haplotypes in the METTL21C gene and peak bone mineral density and body composition in Chinese male nuclear families. J. Bone Miner. Metab. 2017, 35, 437-447. [CrossRef] [PubMed]

13. Chapman, E.; Fry, A.N.; Kang, M. The complexities of p97 function in health and disease. Mol. Biosyst. 2011, 7, 700-710. [CrossRef] [PubMed] 
14. Raasi, S.; Wolf, D.H. Ubiquitin receptors and ERAD: A network of pathways to the proteasome. Semin. Cell Dev. Biol. 2007, 18, 780-791. [CrossRef] [PubMed]

15. Pye, V.E.; Dreveny, I.; Briggs, L.C.; Sands, C.; Beuron, F.; Zhang, X.; Freemont, P.S. Going through the motions: The ATPase cycle of p97. J. Struct. Biol. 2006, 156, 12-28. [CrossRef] [PubMed]

16. Ye, Y. Diverse functions with a common regulator: Ubiquitin takes command of an AAA ATPase. J. Struct. Biol. 2006, 156, 29-40. [CrossRef]

17. Latterich, M.; Fröhlich, K.U.; Schekman, R. Membrane fusion and the cell cycle: Cdc48p participates in the fusion of ER membranes. Cell 1995, 82, 885-893. [CrossRef]

18. Ye, Y.; Meyer, H.H.; Rapoport, T.A. The AAA ATPase Cdc48/p97 and its partners transport proteins from the ER into the cytosol. Nature 2001, 414, 652-656. [CrossRef]

19. Indig, F.E.; Partridge, J.J.; von Kobbe, C.; Aladjem, M.I.; Latterich, M.; Bohr, V.A. Werner syndrome protein directly binds to the AAA ATPase p97/VCP in an ATP-dependent fashion. J. Struct. Biol. 2004, 146, 251-259. [CrossRef]

20. Partridge, J.J.; Lopreiato, J.O.; Latterich, M.; Indig, F.E. DNA damage modulates nucleolar interaction of the Werner protein with the AAA ATPase p97/VCP. Mol. Biol. Cell 2003, 14, 4221-4229. [CrossRef]

21. Watts, G.D.; Wymer, J.; Kovach, M.J.; Mehta, S.G.; Mumm, S.; Darvish, D.; Pestronk, A.; Whyte, M.P.; Kimonis, V.E. Inclusion body myopathy associated with Paget disease of bone and frontotemporal dementia is caused by mutant valosin-containing protein. Nat. Genet. 2004, 36, 377-381. [CrossRef] [PubMed]

22. Weihl, C.C.; Dalal, S.; Pestronk, A.; Hanson, P.I. Inclusion body myopathy-associated mutations in p97/VCP impair endoplasmic reticulum-associated degradation. Hum. Mol. Genet. 2006, 15, 189-199. [CrossRef] [PubMed]

23. Thiele, W.; Novac, N.; Mink, S.; Schreiber, C.; Plaumann, D.; Fritzmann, J.; Cremers, N.; Rothley, M.; Schwager, C.; Regiert, T.; et al. Discovery of a novel tumour metastasis-promoting gene, NVM-1. J. Pathol. 2011, 225, 96-105. [CrossRef] [PubMed]

24. López Hernández, J.F.; Zanders, S.E. Veni, vidi, vici: The success of wtf meiotic drivers in fission yeast. Yeast 2018, 35, 447-453. [CrossRef] [PubMed]

25. Hillier, L.D.; Miller, W.; Birney, E.; Warren, W.; Hardison, R.C.; Ponting, C.P.; Bork, P.; Burt, D.W.; Groenen, M.A.M.; Delany, M.E.; et al. Sequence and comparative analysis of the chicken genome provide unique perspectives on vertebrate evolution. Nature 2004, 432, 695-716.

26. Church, D.M.; Goodstadt, L.; Hillier, L.W.; Zody, M.C.; Goldstein, S.; She, X.; Bult, C.J.; Agarwala, R.; Cherry, J.L.; DiCuccio, M.; et al. Lineage-specific biology revealed by a finished genome assembly of the mouse. PLoS Biol. 2009, 7, e1000112. [CrossRef] [PubMed]

27. Wong, K.H.Y.; Levy-Sakin, M.; Kwok, P.Y. De novo human genome assemblies reveal spectrum of alternative haplotypes in diverse populations. Nat. Commun. 2018, 9, 3040. [CrossRef]

28. Wang, G.M.; Yin, H.; Qiao, X.; Tan, X.; Gu, C.; Wang, B.H.; Cheng, R.; Wang, Y.Z.; Zhang, S.L. F-box genes: Genome-wide expansion, evolution and their contribution to pollen growth in pear (Pyrus bretschneideri). Plant Sci. 2016, 253, 164-175. [CrossRef]

29. Kumar, S.; Nei, M.; Dudley, J.; Tamura, K. MEGA: A biologist-centric software for evolutionary analysis of DNA and protein sequences. Brief. Bioinform. 2008, 9, 299-306. [CrossRef]

30. Kumar, S.; Stecher, G.; Tamura, K. MEGA7: Molecular Evolutionary Genetics Analysis Version 7.0 for Bigger Datasets. Mol. Biol. Evol. 2016, 33, 1870-1874. [CrossRef]

31. Lescot, M.; Déhais, P.; Thijs, G.; Marchal, K.; Moreau, Y.; Van de Peer, Y.; Rouzé, P.; Rombauts, S. PlantCARE, a database of plant cis-acting regulatory elements and a portal to tools for in silico analysis of promoter sequences. Nucleic Acids Res. 2002, 30, 325-327. [CrossRef] [PubMed]

32. Bailey, T.L.; Johnson, J.; Grant, C.E.; Noble, W.S. The MEME Suite. Nucleic Acids Res. 2015, 43, W39-W49. [CrossRef] [PubMed]

33. Hu, Y.; Woeste, K.E.; Zhao, P. Completion of the Chloroplast Genomes of Five Chinese Juglans and Their Contribution to Chloroplast Phylogeny. Front. Plant Sci. 2016, 7, 1955. [CrossRef] [PubMed]

34. Sassa, H.; Kakui, H.; Miyamoto, M.; Suzuki, Y.; Hanada, T.; Ushijima, K.; Kusaba, M.; Hirano, H.; Koba, T. S locus F-box brothers: Multiple and pollen-specific F-box genes with S haplotype-specific polymorphisms in apple and Japanese pear. Genetics 2007, 175, 1869-1881. [CrossRef] [PubMed] 
35. Saeed, A.I.; Sharov, V.; White, J.; Li, J.; Liang, W.; Bhagabati, N.; Braisted, J.; Klapa, M.; Currier, T.; Thiagarajan, M.; et al. TM4: A free, open-source system for microarray data management and analysis. BioTechniques 2003, 34, 374-378. [CrossRef] [PubMed]

36. Deng, W.; Wang, Y.; Liu, Z.; Cheng, H.; Xue, Y. HemI: A toolkit for illustrating heatmaps. PLoS ONE $2014,9$. [CrossRef] [PubMed]

37. Kakui, H.; Kato, M.; Ushijima, K.; Kitaguchi, M.; Kato, S.; Sassa, H. Sequence divergence and loss-of-function phenotypes of $S$ locus F-box brothers genes are consistent with non-self recognition by multiple pollen determinants in self-incompatibility of Japanese pear (Pyrus pyrifolia). Plant J. 2011, 68, 1028-1038. [CrossRef] [PubMed]

38. Grabherr, M.G.; Haas, B.J.; Yassour, M.; Levin, J.Z.; Thompson, D.A.; Amit, I.; Adiconis, X.; Fan, L.; Raychowdhury, R.; Zeng, Q.; et al. Full-length transcriptome assembly from RNA-Seq data without a reference genome. Nat. Biotechnol. 2011, 29, 644-652. [CrossRef] [PubMed]

39. Li, P.; Piao, Y.; Shon, H.S.; Ryu, K.H. Comparing the normalization methods for the differential analysis of Illumina high-throughput RNA-Seq data. BMC Bioinform. 2015, 16, 347. [CrossRef]

40. Maag, J.L.V. Gganatogram: An R package for modular visualisation of anatograms and tissues based on ggplot2. F1000Research 2018, 7, 1576. [CrossRef]

41. Feng, S.; Tan, H.; Ling, H.; Yuan, X. Detecting overexpression level of HER2 gene in NSCLC by real-time quantitative PCR and the 2[-Delta Delta C(T)] method. Chin. J. Lung Cancer 2011, 14, 938-942.

42. Expósito-Rodríguez, M.; Borges, A.A.; Borges-Pérez, A.; Pérez, J.A. Selection of internal control genes for quantitative real-time RT-PCR studies during tomato development process. BMC Plant Biol. 2008, 8, 131. [CrossRef] [PubMed]

43. Petrossian, T.C.; Clarke, S.G. Uncovering the human methyltransferasome. Mol. Cell. Proteom. $2011,10$. [CrossRef] [PubMed]

44. Miao, Y.W.; Peng, M.S.; Wu, G.S.; Ouyang, Y.N.; Yang, Z.Y.; Yu, N.; Liang, J.P.; Pianchou, G.; Beja-Pereira, A.; Mitra, B.; et al. Chicken domestication: An updated perspective based on mitochondrial genomes. Heredity 2013, 110, 277-282. [CrossRef] [PubMed]

45. Burt, D.W.; Carrë, W.; Fell, M.; Law, A.S.; Antin, P.B.; Maglott, D.R.; Weber, J.A.; Schmidt, C.J.; Burgess, S.C.; McCarthy, F.M. The Chicken Gene Nomenclature Committee report. BMC Genom. 2009, 10, S5. [CrossRef] [PubMed]

46. Reiff, R.E.; Ali, B.R.; Baron, B.; Yu, T.W.; Ben-Salem, S.; Coulter, M.E.; Schubert, C.R.; Hill, R.S.; Akawi, N.A.; Al-Younes, B.; et al. METTL23, a transcriptional partner of GABPA, is essential for human cognition. Hum. Mol. Genet. 2014, 23, 3456-3466. [CrossRef] [PubMed]

47. Bernkopf, M.; Webersinke, G.; Tongsook, C.; Koyani, C.N.; Rafiq, M.A.; Ayaz, M.; Müller, D.; Enzinger, C.; Aslam, M.; Naeem, F.; et al. Disruption of the methyltransferase-like 23 gene METTL23 causes mild autosomal recessive intellectual disability. Hum. Mol. Genet. 2014, 23, 4015-4023. [CrossRef]

48. Davydova, E.; Ho, A.Y.Y.; Malecki, J.; Moen, A.; Enserink, J.M.; Jakobsson, M.E.; Loenarz, C.; Falnes, P.Ø. Identification and characterization of a novel evolutionarily conserved lysine-specific methyltransferase targeting eukaryotic translation elongation factor 2 (eEF2). J. Biol. Chem. 2014, 289, 30499-30510. [CrossRef]

49. Kaul, G.; Pattan, G.; Rafeequi, T. Eukaryotic elongation factor-2 (eEF2): Its regulation and peptide chain elongation. Cell Biochem. Funct. 2011, 29. [CrossRef]

50. Cloutier, P.; Lavallée-Adam, M.; Faubert, D.; Blanchette, M.; Coulombe, B. Methylation of the DNA/RNA-binding protein Kin17 by METTL22 affects its association with chromatin. J. Proteom. 2014, 100, 115-124. [CrossRef]

51. Jakobsson, M.E.; Moen, A.; Bousset, L.; Egge-Jacobsen, W.; Kernstock, S.; Melki, R.; Falnes, P.Ø. Identification and characterization of a novel human methyltransferase modulating Hsp70 protein function through lysine methylation. J. Biol. Chem. 2013, 288, 27752-27763. [CrossRef] [PubMed]

52. Freeling, M. Bias in plant gene content following different sorts of duplication: Tandem, whole-genome, segmental, or by transposition. Annu. Rev. Plant Biol. 2009, 60, 433-453. [CrossRef] [PubMed]

53. Gorlova, O.; Fedorov, A.; Logothetis, C.; Amos, C.; Gorlov, I. Genes with a large intronic burden show greater evolutionary conservation on the protein level. BMC Evol. Biol. 2014, 14, 50. [CrossRef] [PubMed]

54. Long, M.; Deutsch, M. Association of intron phases with conservation at splice site sequences and evolution of spliceosomal introns. Mol. Biol. Evol. 1999, 16, 1528-1534. [CrossRef] 
55. Dinant, S.; Clark, A.M.; Zhu, Y.; Vilaine, F.; Palauqui, J.C.; Kusiak, C.; Thompson, G.A. Diversity of the superfamily of phloem lectins (phloem protein 2) in angiosperms. Plant. Physiol. 2003, 131, 114-128. [CrossRef]

56. Bai, C.; Sen, P.; Hofmann, K.; Ma, L.; Goebl, M.; Harper, J.W.; Elledge, S.J. SKP1 connects cell cycle regulators to the ubiquitin proteolysis machinery through a novel motif, the F-box. Cell 1996, 86, 263-274. [CrossRef] 